\title{
Detecting scale anomaly in chiral phase transition of QCD: new critical endpoint pinned down
}

\author{
Mamiya Kawaguchi, ${ }^{a, b}$ Shinya Matsuzaki ${ }^{c}$ and Akio Tomiya ${ }^{d, e}$ \\ ${ }^{a}$ Department of Physics and Center for Field Theory and Particle Physics, Fudan University, \\ 220 Handan Road, 200433 Shanghai, China \\ ${ }^{b}$ School of Nuclear Science and Technology, University of Chinese Academy of Sciences, \\ Beijing 100049, China \\ ${ }^{c}$ Center for Theoretical Physics and College of Physics, Jilin University, \\ Changchun, 130012, China \\ ${ }^{d}$ RIKEN BNL Research center, Brookhaven National Laboratory, \\ Upton, NY, 11973, U.S.A. \\ ${ }^{e}$ Department of Information Technology, \\ International Professional University of Technology in Osaka, \\ 3-3-1 Umeda, Kita-Ku, Osaka, 530-0001, Japan
}

E-mail: kawaguchi@fudan.edu.cn, synya@jlu.edu.cn, akio.tomiya@riken.jp

ABSTRACT: Violation of scale symmetry, scale anomaly, being a radical concept in quantum field theory, is of importance to comprehend the vacuum structure of QCD, and should potentially contribute to the chiral phase transition in thermal QCD, as well as the chiral and U(1) axial symmetry. Though it should be essential, direct evidence of scale anomalies has never been observed in the chiral phase transition. We propose a methodology to detect a scale anomaly in the chiral phase transition, which is an electromagnetically induced scale anomaly: apply a weak magnetic field background onto two-flavor massless QCD with an extremely heavy strange quark, first observe the chiral crossover; second, adjusting the strange quark mass to be smaller and smaller, observe the second-order chiral phase transition, and then the first-order one in the massless-three flavor limit. Thus, the second-order chiral phase transition, observed as the evidence of the quantum scale anomaly, is a new critical endpoint. It turns out that this electromagnetic scale anomaly gets most operative in the weak magnetic field regime, rather than a strong field region. We also briefly address accessibility of lattice QCD, a prospected application to dense matter system, and implications to astrophysical observations, such as gravitational wave productions provided from thermomagnetic QCD-like theories.

Keywords: Anomalies in Field and String Theories, Chiral Lagrangians, Conformal and W Symmetry, Phase Diagram of QCD

ArXiv ePrint: 2102.05294 


\section{Contents}

1 Introduction 1

2 Ginzburg-Landau description $\quad 4$

3 Scale anomaly coupled to chiral order parameter $\quad 6$

3.1 Ward-Takahashi identity for scale symmetry in electromagnetic field 7

3.2 Electromagnetic scale-anomaly induced-tadpole at finite temperature 8

4 A new critical endpoint: demonstration based on quark meson model in the large $N_{c}$ limit $\quad 12$

5 Conclusion and discussion $\quad 15$

$\begin{array}{ll}\text { A Meson masses in linear sigma model } & 17\end{array}$

$\begin{array}{ll}\text { B Chiral phase transition in three flavor symmetric limit } & 18\end{array}$

\section{Introduction}

The origin of scales in QCD can be classified into two categories in terms of quantum field theory: one is spontaneous symmetry breaking of chiral symmetry, while the other explicit symmetry breaking. The latter is subject to renormalization of the quantum corrections in QCD, quantum scale anomalies, and the presence of quark masses (classical- chiral and scale breaking). Applying QCD in a thermal bath would have a potential to detect the direct consequence of the spontaneous breaking of the chiral symmetry, by observing the damping of the order parameter for the chiral phase transition (i.e. the quark condensate) at high temperatures above the critical temperature. Thus, the scale breaking associated with the spontaneous breaking can be dropped out there. However, there is still inevitable contamination left: that is the scale anomaly arising from renormalization evolution of the QCD gauge coupling, which should be present all the way in the thermal history of QCD, and it still coexists with the scale breaking from quark masses, even above the critical temperature. Hence detection of the scale anomaly seems to be quite challenging even in the thermal QCD.

To reconcile this dilemma, it would be necessary to go away, from the physical point, at the massless limit (so-called the chiral limit). There quarks enjoy the exact chiral symmetry and the scale symmetry breaking is provided purely by the quantum scale anomaly. However, the dimensional transmutation in the infrared energy regime, associated with the scale anomaly, triggers the spontaneous chiral breaking, hence contaminates with each 


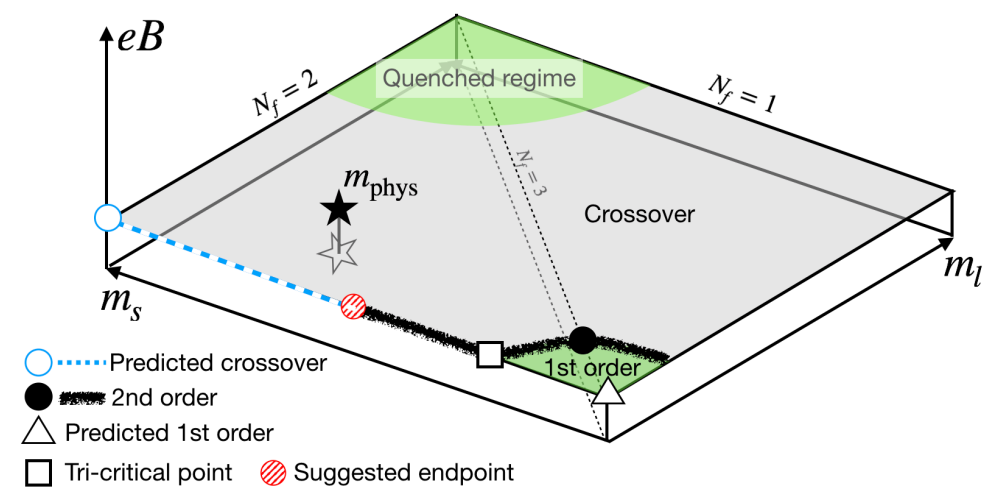

Figure 1. Extended Columbia plot along with an external magnetic field in the weak field regime. $m_{l}$ and $m_{s}$ denote masses of the light quarks and strange quark, respectively. The existence of the scale anomaly is dictated as the suggested endpoint on this Columbia plot, indicated by a shaded circle. A blank square stands for the tri-critical point, at which the three domains of crossover, first- and second-order phase transitions merge. The details are described in the text. The physical point is known to be the crossover [1] at this size of magnetic field.

other in the thermal evolution of the chiral order parameter. Thus the case would still be involved even in the chiral limit.

Pisarski and Wilczek a long time ago [2] payed their particular attention to the chiral limit, off the physical point, and employed an effective model in the confinement phase based on the exact chiral symmetry with or without U(1) axial symmetry. They discussed the renormalization group runnings of the model couplings, and existence of the renormalization group-fixed point. It was suggested that the order of the chiral phase transition highly depends on the number of massless flavors (namely the chiral symmetry and U(1) axial symmetry). This was the pioneering work and made a significant help to comprehend QCD at the physical point as the chiral theory. However, at this point, it is yet unclear how the scale anomaly directly affects the chiral phase transition.

Columbia university group extended their work using lattice QCD calculations [3]. In lattice QCD, mass and the number of flavor dependence on the order of the QCD phase transition have been investigated including the chiral and confinement-deconfinement phase transitions. It is summarized as what is called Columbia plot, which is drawn on the quark mass plane, hence dictates the chiral and U(1) axial symmetry structures.

Since the early work of Columbia group used an approximated algorithm, it has nowadays been refined by utilizing modern technologies. In the two flavor chiral limit and $2+1$ flavor chiral limit, namely with two of massless quarks and one heavy quark, restoration or persistence of $\mathrm{U}(1)$ axial anomaly above the critical temperature has been discussed [4-11]. It is shown that restoration or persistence is tightly connected to the order of phase transition and its universality class [2,12-14]. On the other hand, QCD is believed to exhibit the first-order phase-transition in the three-flavor chiral limit [2]. However, no clear lattice 
results for the first-order phase-transition has been obtained and only lower limits on the quark mass in the crossover has been placed $[15,16]$.

Thus the chiral properties in the chiral phase transition have extensively been studied on lattice QCD and so far been well understood through projection on to the Columbia plot, initiated from the Pisarski-Wilczek's work. However, never have the scale anomaly been clearly understood or given any definite signal even on the Columbia plot: the form of the QCD scale anomaly is flavor universal, hence would survive all the way on the Columbia plot. Thus it may be conclusive that the QCD scale anomaly leaves no distinct footprint on the Columbia plot.

In this paper, we propose a methodology to detect another scale anomaly in the chiral phase transition on a Columbia plot: apply a weak magnetic field background onto QCD, and see that an electromagnetically induced scale anomaly arises, which is coupled to the chiral order parameter; work in two-flavor massless QCD with an extremely heavy strange quark; first observe the chiral crossover; second, adjusting the strange quark mass to be smaller and smaller, observes the second-order chiral phase transition, and the first-order one in the massless-three flavor limit. See figure 1.

That is an evidence of the quantum scale anomaly (an electromagnetically induced quantum scale anomaly), which turns to transparently contribute to the chiral phase transition, so is the essential key to significantly affect the Columbia plot, as well as the chiral and $\mathrm{U}(1)$ axial symmetries. It turns out that this electromagnetic scale anomaly gets most operative in the weak magnetic field regime. In the massless-three flavor limit, this quantum scale anomaly contribution is overwhelmed by the U(1) axial anomaly's, hence the first-order phase transition is realized, while in the massless-two flavor limit such interference does not happen, and the crossover is promoted by the induced tadpole, as will explicitly be demonstrated later. Thus, the second-order chiral phase transition, observed as the evidence of the quantum scale anomaly, is a new critical endpoint.

Other critical points have been proposed in a strong magnetic field regime [17, 18]. Our finding is definitely different from those which are expected to emerge due to broadening of the quenched regime in figure 1 because of the amplified mass gap of quarks by the strong magnetic field.

Lots of interesting results have been reported from lattice studies on the QCD thermodynamics in the strong external magnetic field [19-22]. Of these results, there are two striking properties discovered: the reduction of transition temperature [23-27] and the inverse magnetic catalysis [1]. In contrast to the strong magnetic field the weak magnetic field has not fully been investigated on the lattice QCD at non-zero temperature. At the current status, the smallest nonzero magnetic field on the lattice is $\sqrt{e B}_{\min } \sim 0.1 \mathrm{GeV}[1]$, which is only for the physical pion mass simulations at finite temperatures. ${ }^{1}$ This is because of the fact that the minimal magnetic field is determined by the area of transverse plane to the magnetic field [1]. Thus the weak magnetic field with smaller pion masses, particularly close to the chiral limit, is challenging due to the higher numerical cost.

\footnotetext{
${ }^{1}$ On the lattice, the minimum of magnetic field is bounded by the temperature, $|e B|_{\min } \propto T^{2}$. See [1] for details.
} 
Instead of lattices, chiral effective theories, like what we will employ, would therefore shed light on the weak magnetic regime at the chiral limit, as if it gets back to the epoch where the Columbia plot was first proposed along the pioneering work by Pisarski and Wilczek: our finding paves a way to investigate this new frontier as searching for the evidence of the quantum scale anomaly in the chiral phase transition.

The proposed critical endpoint is marked as "Suggested endpoint", in an extended Columbia plot including the magnetic field in the weak field regime, figure 1 . The new endpoint reveals the potential existence of the scale anomaly in QCD, which is made transparent in the presence of a magnetic field. This is our main result in the present paper.

\section{Ginzburg-Landau description}

Before entering the detailed demonstration, we shall present an intuitive interpretation on the chiral phase transition nature, which would help readers to easily grasp our new finding. To this end, we momentarily work on a generic Ginzburg-Landau description for the chiral phase transition, inspired by Pisarski and Wilczek. ${ }^{2}$

In the Ginzburg-Landau approach, the chiral phase structure can be described by a generic effective potential in terms of the order parameter $\bar{\sigma}_{0}$. It takes the polynomial form.

$$
\begin{aligned}
V_{\text {eff }}\left(\bar{\sigma}_{0}\right)= & \alpha_{1}(T, e B) \bar{\sigma}_{0}+\alpha_{2}(T, e B) \bar{\sigma}_{0}^{2} \\
& +\alpha_{3}(T, e B) \bar{\sigma}_{0}^{3}+\alpha_{4}(T, e B) \bar{\sigma}_{0}^{4}+\cdots
\end{aligned}
$$

where the potential parameters $\alpha_{i=1,2,3,4, \ldots}$ can include intrinsic temperature - and/or magnetic - dependence. Our sigma field is defined as an interpolating mesonic degree of freedom for the lightest quark bilinear $\bar{q} q$ in QCD.

When the exact chiral symmetry is imposed, the effective potential form is restricted to be an even function of $\bar{\sigma}_{0}$, such as $\bar{\sigma}_{0}^{2}$ and $\bar{\sigma}_{0}^{4}$. With terms truncated up to $O\left(\bar{\sigma}_{0}^{4}\right)$, the phase transition is then expected to be of the second order.

In the three-flavor case, the $\mathrm{U}(1)$ axial anomaly induces the cubic term of $\bar{\sigma}_{0}$, which originally takes a determinant form of the quark condensate, $\operatorname{det} \bar{q} q \sim \bar{\sigma}_{0}^{3}$, a la KobayashiMaskawa-'t Hooft [28-31]. Then, the phase transition order is changed to be of the first order, because the cubic term creates a potential barrier between the chiral symmetric vacuum $\left(\bar{\sigma}_{0}=0\right)$ and broken one $\left(\bar{\sigma}_{0} \neq 0\right)$. Note here that the determinant term in the two-flavor case can be absorbed into $\bar{\sigma}_{0}^{2}$ term, so the U(1) axial anomaly does not effectively affect the potential form in eq. (2.1).

As to the linear (tadpole) term of $\bar{\sigma}_{0}$, it cannot be present even with the $\mathrm{U}(1)$ axial anomaly. It would show up when the current quark masses are introduced, which can explicitly break the full chiral symmetry including the U(1) axial part. Once the tadpole is present, the potential no longer achieves the exactly chiral symmetric vacuum, which, in other words, the theory always lies in the broken phase, even though the value of $\bar{\sigma}_{0}$ gets

\footnotetext{
${ }^{2}$ Our current analysis is based on an effective potential approach, not on the renormalization group method like in the Pisarski and Wilczek's work. Cross-check using the (nonperturbative) renormalization group would make our finding more evident, which will be pursued elsewhere.
} 
smaller, as $T$ becomes higher. This implies that the tadpole term in the potential tends to drive the phase transition to be continuous, i.e., crossover, called the chiral crossover.

To make the point better understood, we may explicitly introduce the chiral field $\Phi$, which is parametrized by the scalar- and pseudoscalar-meson fields as

$$
\Phi= \begin{cases}\frac{1}{2}\left(\sigma_{0}+i \tau^{i} \pi^{i}\right), & \left(\text { for } N_{f}=2\right) \\ \frac{1}{2}\left(\sigma_{a}+i \pi_{a}\right) \lambda^{a}, & \left(\text { for } N_{f}=3\right),\end{cases}
$$

where $\tau_{i=1, \cdots, 3}$ are the Pauli matrices and $\lambda_{a=1, \cdots, 8}$ represent the Gell-Mann matrices with $\lambda_{0}=\sqrt{2 / 3} \cdot \mathbf{1}_{3 \times 3}$. Under the chiral symmetry we define the transformation law of $\Phi$ as

$$
\Phi \rightarrow\left\{\begin{array}{l}
g_{L} \cdot \Phi \cdot g_{R}^{\dagger}, \quad\left(\text { for } N_{f}=2\right) \\
g_{A} \cdot g_{L} \cdot \Phi \cdot g_{R}^{\dagger}, \quad\left(\text { for } N_{f}=3\right),
\end{array}\right.
$$

where $g_{L, R} \in \mathrm{SU}(2)_{L, R}$ in the case of $N_{f}=2$, and $g_{L, R} \in \mathrm{SU}(3)_{L, R}$ with $g_{A} \in \mathrm{U}(1)_{A}$ in the case of $N_{f}=3$.

Thus the linear sigma model as the Ginzburg-Landau description is constructed based on the chiral invariance with its breaking source, including operators up to dimension four, as

$$
\mathcal{L}_{\mathrm{LSM}}=\operatorname{tr}\left[\partial_{\mu} \Phi \partial^{\mu} \Phi^{\dagger}\right]-V(\Phi),
$$

where $V(\Phi)$ represents the potential terms,

$$
V(\Phi)=V_{0}(\Phi)+V_{\text {anom }}(\Phi)+V_{\mathrm{SB}}(\Phi) .
$$

In eq. (2.5) $V_{0}(\Phi)$ is the chiral invariant part:

$$
V_{0}(\Phi)=\mu^{2} \operatorname{tr}\left[\left(\Phi^{\dagger} \Phi\right)\right]+\lambda_{1} \operatorname{tr}\left[\left(\Phi^{\dagger} \Phi\right)^{2}\right]+\lambda_{2}\left(\operatorname{tr}\left[\left(\Phi^{\dagger} \Phi\right)\right]\right)^{2},
$$

where $\mu^{2}$ is the mass parameter taken either a positive or negative value. $\lambda_{1,2}$ are dimensionless quartic coupling constants, which cannot be taken independently in the case of $N_{f}=2$, because of the speciality of $\mathrm{SU}(2)$ algebra.

For $N_{f}=3$, the $\mathrm{U}(1)$ axial anomalous part, but keeping the chiral $\mathrm{SU}\left(N_{f}\right)_{L} \times \mathrm{SU}\left(N_{f}\right)_{R}$ symmetry, represents $V_{\text {anom }}(\Phi)$. The lowest dimensional operator for the U(1) axial anomalous part is given by a la Kobayashi-Maskawa-'t Hooft [28-31],

$$
V_{\text {anom }}(\Phi)=-B_{\text {anom }}\left(\operatorname{det}[\Phi]+\operatorname{det}\left[\Phi^{\dagger}\right]\right),
$$

where the parameter $B_{\text {anom }}$ is taken to be real, which has mass dimension one.

In the case of $N_{f}=2, V_{\text {anom }}$ is redundant: $\operatorname{det} \Phi+$ h.c. $=2\left(\sigma_{0}^{2}+\left(\pi_{i}\right)^{2}\right)$ hence is indistinguishable from the $\mu^{2}$ term, because $\Phi$ has no $\mathrm{U}(1)_{A}$ charge as in eq. (2.3).

In the underlying QCD Lagrangian, the chiral symmetry is explicitly broken by the current quark mass matrix $\mathcal{M}=\operatorname{diag}\left\{m_{u}, m_{d}\left(, m_{s}\right)\right\}$. In the linear-sigma model-Lagrangian, this explicit breaking effect is reflected in the $V_{\mathrm{SB}}$ part:

$$
V_{\mathrm{SB}}(\Phi)=-c \operatorname{tr}\left[\mathcal{M} \Phi^{\dagger}+\mathcal{M}^{\dagger} \Phi\right]
$$

where the parameter $c$ is taken to be a real value, and has mass dimension two. 
To be consistent with the underlying QCD, we choose the background-field profile for the $\Phi$ field to respect the vectorial symmetry, $\mathrm{SU}(2)_{V}$ or $\mathrm{SU}(3)_{V}$, in such a way that

$$
\bar{\Phi} \cdot \mathbf{1}=\left\{\begin{array}{l}
\frac{1}{2} \bar{\sigma}_{0} \cdot \mathbf{1}_{2 \times 2}, \quad\left(\text { for } N_{f}=2\right) \\
\frac{1}{2} \sqrt{\frac{2}{3}} \bar{\sigma}_{0} \cdot \mathbf{1}_{3 \times 3}, \quad\left(\text { for } N_{f}=3\right) .
\end{array}\right.
$$

Thus the linear sigma model potential can be viewed as a Ginzburg-Landau description, at the tree level (i.e. mean field level):

$$
V_{\text {tree }}\left(\bar{\sigma}_{0}\right)=\left\{\begin{array}{l}
-\sum_{f} c m_{f} \bar{\sigma}_{0}+\frac{1}{2}\left(\mu^{2}-B_{\text {anom }}\right) \bar{\sigma}_{0}^{2}+\left(\frac{\lambda_{1}}{8}+\frac{\lambda_{2}}{4}\right) \bar{\sigma}_{0}^{4}, \quad\left(\text { for } \quad N_{f}=2\right) \\
-\sqrt{\frac{2}{3}} \sum_{f} c m_{f} \bar{\sigma}_{0}+\frac{1}{2} \mu^{2} \bar{\sigma}_{0}^{2}-\frac{B_{\text {anom }}}{3 \sqrt{6}} \bar{\sigma}_{0}^{3}+\left(\frac{\lambda_{1}}{12}+\frac{\lambda_{2}}{4}\right) \bar{\sigma}_{0}^{4}, \quad\left(\text { for } \quad N_{f}=3\right) .
\end{array}\right.
$$

From this potential, the vacuum expectation value $\left\langle\bar{\sigma}_{0}\right\rangle$ can be determined from the stationary condition,

$$
\left.\frac{\partial V_{\text {eff }}\left(\bar{\sigma}_{0}\right)}{\partial \bar{\sigma}_{0}}\right|_{\bar{\sigma}_{0}=\left\langle\bar{\sigma}_{0}\right\rangle=\sqrt{2 N_{f}}\langle\bar{\Phi}\rangle}=0
$$

At the tree level evaluation, it is related to the pion decay constant $f_{\pi}$ as

$$
\langle\bar{\Phi}\rangle=\frac{1}{\sqrt{2 N_{f}}}\left\langle\bar{\sigma}_{0}\right\rangle=f_{\pi} / 2
$$

We can also discuss the order of chiral phase transition from the tree-level potential in eq. (2.10). At the chiral limit for the case of $N_{f}=2\left(m_{f}=0\right)$, the parameter $B_{\text {anom }}$ can be absorbed in the mass parameter $\mu^{2}$, so that the $\mathrm{U}(1)$ axial anomaly part does not effectively affect the order of chiral phase transition. The tree-level potential then has only the $\bar{\sigma}_{0}^{2}$ and $\bar{\sigma}_{0}^{4}$ terms. Thus, in the massless two-flavor case, the phase transition is deduced to be of the second order.

In the massless three-flavor case, the U(1) axial anomaly part generates the cubic term of $\bar{\sigma}_{0}$, which creates a potential barrier between the chiral symmetric vacuum $\left(\bar{\sigma}_{0}=0\right)$ and broken one $\left(\bar{\sigma}_{0} \neq 0\right)$. In association with the deformation of the potential, the phase transition is changed to be of the first order.

We will later observe that a tadpole term is generated by the electromagnetic scale anomaly, even for the massless flavor cases, in the thermomagnetic QCD. The phase transition becomes crossover, so there should be a critical endpoint seen in the interplay between massless two-flavor and three-flavor thermomagnetic QCD, as in figure 1.

\section{Scale anomaly coupled to chiral order parameter}

In this section we discuss how the chiral order parameter can affect the scale anomaly. First, note that in the low-energy meson-dynamics, the scalar meson fields serve as a source for the scale symmetry breaking in QCD, accompanied with the chiral symmetry breaking. Then the scale (dilatation) current $j_{D}(x)$ is composed of hadrons, and would couple to the chiral singlet/isosinglet states, involving the (chiral singlet/isosinglet component of) $\sigma$ meson state, the two-pion resonant state, a tetraquark state, and glueball, and so forth. 
Since we work on the chiral phase transition by the Ginzburg-Landau description and are interested particularly in a coupling of electromagnetic field background to the chiral order parameter, only the chiral singlet/isosinglet component of the meson field is relevant.

We also assume the lightest isoscalar mesons to be mostly composed of quarkonium state, so that the vacuum expectation value associated with the tetraquark state is negligible. One might think that a glueball field can mix with the sigma meson field, hence might contribute to the scale anomaly relevant to the chiral phase transition. However, it has recently been reported from lattice simulations for $2+1$ flavors that around the chiral limit which is currently our main concern, the fluctuation of Polyakov loop, which can be regarded as (an electric part) of glueball, does not have significant correlation with the chiral order parameter [32]. This indicates negligible mixing between the glueball and sigma meson around the chiral limit (with mass less than the physical-point value). Hence such a gluonic term will not be taken into account in the present work.

Thus, as far as the coupling to the chiral order parameter at the low-energy is concerned, we can approximate the overlap amplitude between the scale current and (the chiral singlet/isosinglet component of) the sigma meson state, dubbed $\phi$, as $^{3}$

$$
\left\langle 0\left|\partial_{\mu} j_{D}^{\mu}(x)\right| \phi(p)\right\rangle=\left\langle 0\left|\left(T_{\mu}^{\mu}\right)_{\text {meson }}(x)\right| \phi(p)\right\rangle \approx f_{\phi} m_{\phi}^{2} e^{-i p \cdot x},
$$

where we have taken $\langle\phi\rangle=f_{\phi}$, which plays the role of the decay constant for the scale breaking, and $\left(T^{\mu}\right)_{\text {meson }}$ is the trace of the energy momentum tensor described by mesons.

\subsection{Ward-Takahashi identity for scale symmetry in electromagnetic field}

The scale symmetry is also explicitly broken by the electromagnetic contribution and becomes anomalous. As a consequence, the chiral-singlet/isosinglet scalar can be coupled to the electromagnetic field via the scale anomaly arising from the quantum correction of the quark loop. To obtain the coupling form between the scalar field and the electromagnetic field, we consider the correlation function related to the Ward-Takahashi identity for a photon two-point function coupled with the dilatation current $j_{D}^{\mu}$,

$$
\lim _{q \rightarrow 0} \int d^{4} y e^{i q \cdot y}\left\langle 0\left|T \partial_{\rho} j_{D}^{\rho}(y) A_{\mu}(x) A_{\nu}(0)\right| 0\right\rangle=i \delta_{D}\left\langle 0\left|T A_{\mu}(x) A_{\nu}(0)\right| 0\right\rangle,
$$

where $\delta_{D}$ denotes the infinitesimal scale transformation by the charge $Q_{D}=\int d^{3} \vec{x} j_{D}^{0}(x)$, defined as $\left[i Q_{D}, \mathcal{O}(x)\right]=\delta_{D} \mathcal{O}(x)=\left(d_{\mathcal{O}}+x^{\nu} \partial_{\nu}\right) \mathcal{O}(x)$, for an operator $\mathcal{O}$ with the scaling dimension $d_{\mathcal{O}}$.

Assuming the lightest scalar-meson pole-dominance, we can rewrite the left-hand side of eq. (3.2),

$$
\lim _{q \rightarrow 0} \int d^{4} y e^{i q \cdot y}\left\langle 0\left|T \partial_{\rho} j_{D}^{\rho}(y) A_{\mu}(x) A_{\nu}(0)\right| 0\right\rangle=-i f_{\phi} \lim _{q \rightarrow 0}\left\langle\phi(q)\left|T A_{\mu}(x) A_{\nu}(0)\right| 0\right\rangle .
$$

Here, we have used eq. (3.1). Hence, the overlap amplitude of two photons with the chiral-singlet/isosinglet scalar state can be described by the scale transformation for the

\footnotetext{
${ }^{3}$ In eq. (3.1), the overall sign can be minus, and then it can be absorbed in the decay constant $f_{\phi}$.
} 
photon propagator,

$$
\left\langle\phi(q=0)\left|T A_{\mu}(x) A_{\nu}(0)\right| 0\right\rangle=-\frac{1}{f_{\phi}} \delta_{D}\left\langle 0\left|T A_{\mu}(x) A_{\nu}(0)\right| 0\right\rangle .
$$

By using eq. (3.4), later we will find the coupling form between the sigma meson field and the electromagnetic field.

\subsection{Electromagnetic scale-anomaly induced-tadpole at finite temperature}

In the magnetized-thermal bath, the Lorentz invariance in four dimensions is lost. Then, the photon propagator expression should be no longer Lorentz or $O(3)$ covariant, due to the quark-loops in the bath, which would yield intrinsic magnetic corrections to the photon polarization function, as well as the thermal corrections. Note, however, that our current main concern is the overlap amplitude between the $\phi$ scalar and two photon fields in eq. (3.4), which, in terms of the interaction Lagrangian, should take the form like $\sim f(e B, T) \bar{\phi} F_{\mu \nu} F^{\mu \nu} \sim f(e B, T) \bar{\phi} B^{2}$, where $\bar{\phi}$ is the background field of $\phi$, and $f(e B, T)$ is an associated form factor arising from the thermomagnetized quark loop corrections. Therefore, in the weak magnetic regime that involves our current concern, we can ignore the $e B$-dependence in $f(e B, T)$, because it would give subleading corrections like $\mathcal{O}\left((e B)^{4}\right)$ to the total $\phi$-photon-photon amplitude. Hence, as far as the weak magnetic field regime is concerned, the magnetic field dependence on the photon propagator involving polarization functions like $f(e B, T)$ can be ignored. We will further give comments on this higher order correction later (in Conclusion and Discussion section).

In that case, the photon propagator only gets the thermal quantum correction to the quark-photon vertex with a (dynamical) quark mass $m_{\mathrm{dyn}}$. Then the polarization structure can be generally decomposed into the three independent components,

$$
D_{\mu \nu}(K)=\frac{-i}{K^{2}-\Pi_{T}} P_{\mu \nu}+\frac{-i}{K^{2}-\Pi_{L}} Q_{\mu \nu}+(\xi-1) \frac{i K_{\mu} K_{\nu}}{K^{4}},
$$

where the four-momentum of the photon denotes $K_{\mu}=\left(\omega, k_{1}, k_{2}, k_{3}\right) . \xi$ is the gauge fixing parameter, and $P_{\mu \nu}$ and $Q_{\mu \nu}$ represent the transverse- and the longitudinal-polarization tensors,

$$
\begin{aligned}
P_{\mu \nu} & =\tilde{\eta}_{\mu \nu}+\frac{\tilde{K}_{\mu} \tilde{K}_{\nu}}{\vec{k}^{2}}, \\
Q_{\mu \nu} & =\frac{-1}{K^{2} \vec{k}^{2}}\left(\vec{k}^{2} u_{\mu}+\omega \tilde{K}_{\mu}\right)\left(\vec{k}^{2} u_{\nu}+\omega \tilde{K}_{\nu}\right),
\end{aligned}
$$

with $\tilde{\eta}_{\mu \nu}, \tilde{K}_{\mu}$ and $u_{\mu}$ being

$$
\begin{aligned}
\tilde{\eta}_{\mu \nu} & =\operatorname{diag}[0,-1,-1,-1], \\
\tilde{K}_{\mu} & =K_{\mu}-\omega u_{\mu}=\left(0, k_{1}, k_{2}, k_{3}\right), \\
u_{\mu} & ==(+1,0,0,0) .
\end{aligned}
$$

The loop corrections $\Pi_{T, L}$ separately include the vacuum part $\Pi_{T, L}^{(T=0)}$ and thermal $\operatorname{part} \Pi_{T, L}^{(T \neq 0)}$

$$
\Pi_{T, L}=\Pi_{T, L}^{(T=0)}+\Pi_{T, L}^{(T \neq 0)}
$$


At the quark one-loop calculation performed by the dimensional regularization in $4-\epsilon$ dimension, $\Pi_{T, L}$ are expressed as (for the detail of the thermal part, see [33, 34])

$$
\begin{aligned}
\Pi_{T}^{(T=0)}= & \Pi_{L}^{(T=0)}=-\frac{N_{c}}{2 \pi^{2}} \sum_{f} Q_{f}^{2} K^{2} \int_{0}^{1} d x x(1-x)\left(\frac{2}{\epsilon}-\log \left\{m_{\mathrm{dyn}}^{2}-x(1-x) K^{2}\right\}-\gamma+\log (4 \pi)\right) \\
\Pi_{T}^{(T \neq 0)=} & \frac{2 N_{c}}{\pi^{2}} \sum_{f} Q_{f}^{2}\left[\left\{\frac{\omega^{2}}{\vec{k}^{2}}+\left(1-\frac{\omega^{2}}{\vec{k}^{2}}\right) \ln \frac{\omega+|\vec{k}|}{\omega-|\vec{k}|}\right\}\right. \\
& \times\left(m_{\mathrm{dyn}} T \tilde{a}\left(m_{\mathrm{dyn}} / T\right)-T^{2} \tilde{c}\left(m_{\mathrm{dyn}} / T\right)\right) \\
& \left.+\frac{1}{8}\left\{2 m_{\mathrm{dyn}}^{2}+\omega^{2}+\frac{107 \omega^{2}-131 \vec{k}^{2}}{72}\right\} \tilde{b}\left(m_{\mathrm{dyn}} / T\right)\right] \\
\Pi_{L}^{(T \neq 0)}= & \frac{4 N_{c}}{\pi^{2}} \sum_{f} Q_{f}^{2}\left(1-\frac{\omega^{2}}{\vec{k}^{2}}\right)\left[\left(1-\frac{\omega}{2|\vec{k}|} \ln \frac{\omega+|\vec{k}|}{\omega-|\vec{k}|}\right)\left(m_{\mathrm{dyn}} T \tilde{a}\left(m_{\mathrm{dyn}} / T\right)-T^{2} \tilde{c}\left(m_{\mathrm{dyn}} / T\right)\right)\right. \\
& \left.+\frac{1}{4}\left\{2 m_{\mathrm{dyn}}^{2}-\omega^{2}+\frac{11 \vec{k}^{2}+37 \omega^{2}}{72}\right\} \tilde{b}\left(m_{\mathrm{dyn}} / T\right)\right],
\end{aligned}
$$

where $Q_{f}$ with $f=u, d$ (and $s$ ) is the electromagnetic charge for quark flavor $f, Q_{u}=$ $2 e / 3, \quad Q_{d}=-e / 3, \quad Q_{s}=-e / 3 ; m_{\mathrm{dyn}}$ is the dynamical quark mass generated by the spontaneous chiral symmetry breaking; $\gamma$ is the Euler-Mascheroni constant. Here, we have taken the flavor universal limit; $m_{\mathrm{dyn}}^{(u)}=m_{\mathrm{dyn}}^{(d)}=m_{\mathrm{dyn}}^{(s)}=m_{\mathrm{dyn}}$, which turns out to be justified in the present framework of analysis even in the presence of the chiral-and isospinbreaking magnetic field (See the later discussion around eq. (4.1)). $\tilde{a}\left(m_{\mathrm{dyn}} / T\right), \tilde{b}\left(m_{\mathrm{dyn}} / T\right)$ and $\tilde{c}\left(m_{\mathrm{dyn}} / T\right)$ are given by

$$
\begin{aligned}
& \tilde{a}\left(m_{\mathrm{dyn}} / T\right)=\ln \left(1+e^{-m_{\mathrm{dyn}} / T}\right), \\
& \tilde{b}\left(m_{\mathrm{dyn}} / T\right)=\sum_{n=1}(-1)^{n} \operatorname{Ei}\left(-n m_{\mathrm{dyn}} / T\right)=\sum_{n=1}(-1)^{n}\left(-\int_{n m_{\mathrm{dyn}} / T}^{\infty} \frac{e^{-t}}{t} d t\right), \\
& \tilde{c}\left(m_{\mathrm{dyn}} / T\right)=\sum_{n=1}^{\infty}(-1)^{n} \frac{e^{-n m_{\mathrm{dyn}} / T}}{n^{2}} .
\end{aligned}
$$

With the above explicit expression, the scale transformation for the photon propagator showing up in eq. (3.4) is evaluated as

$$
\begin{aligned}
\delta_{D} D_{\mu \nu}(x)= & -\int \frac{d^{4} p}{(2 \pi)^{4}}\left[\left\{F^{(T=0)}(K)+F_{T}^{(T \neq 0)}(\omega, \vec{k})\right\} \frac{i}{\left(K^{2}-\Pi_{T}\right)^{2}} P_{\mu \nu}\right. \\
& \left.+\left\{F^{(T=0)}(K)+F_{L}^{(T \neq 0)}(\omega, \vec{k})\right\} \frac{i}{\left(K^{2}-\Pi_{L}\right)^{2}} Q_{\mu \nu}\right] e^{-i K \cdot x},
\end{aligned}
$$

where $F^{(T=0)}(K)$ and $F_{T, L}^{(T \neq 0)}(\omega, \vec{k})$ represent the variation for the vacuum- and the thermalcorrection parts, respectively. The vacuum part $F^{(T=0)}(K)$ is evaluated as

$$
F^{(T=0)}(K)=-2 \frac{\beta(e)}{e} K^{2}+F_{\mathrm{NL}}^{(T=0)}(K)
$$


where

$$
F_{\mathrm{NL}}^{(T=0)}(K)=\frac{N_{c}}{\pi^{2}} \sum_{f} Q_{f}^{2} m_{\mathrm{dyn}}^{2}\left[-1+\int_{0}^{1} d x \frac{1}{1-x(1-x)\left(K^{2} / m_{\mathrm{dyn}}^{2}\right)}\right]
$$

$\beta(e)$ denotes the beta function of the electromagnetic gauge coupling $(e)$, defined as $\beta(e)=$ $\partial e(\mu) / \partial \ln \mu$ with the renormalization scale $\mu$. We evaluate the beta function $\beta(e)$ at the one-loop level of QED as $\beta(e)=\frac{e}{(4 \pi)^{2}} \frac{4 N_{c}}{3} \sum_{f} Q_{f}^{2}$, with $N_{c}=3$. The first term of the right hand side in eq. (3.12) induces the local-tadpole interaction between the $\phi$ and the electromagnetic fields, while the second term $F_{\mathrm{NL}}^{(T=0)}(K)$ corresponds to the nonlocal effective interaction.

The thermal-correction part $F_{T, L}^{(T \neq 0)}(\omega, \vec{k})$ in eq. (3.11) gives the effective interaction between the $\phi$ and the electromagnetic field, which is expressed as the nonlocal form. Note, however, that $F_{L}^{(T \neq 0)}(\omega, \vec{k})$ comes along with the longitudinal polarization of photon, which does not couple to a magnetic field, so that it does not contribute to the thermomagnetic tadpole. Hence this part will be discarded in the present study.

From eq. (3.4) and eq. (3.11), we can read off the effective interaction between the $\phi$ and the electromagnetic field at finite temperature:

$$
\begin{aligned}
\int d^{4} x \mathcal{L}_{\text {int }}^{(\mathrm{Tad})}= & \int d^{4} x \frac{\beta(e)}{2 e} \frac{\phi}{f_{\phi}} F_{\mu \nu} F^{\mu \nu} \\
& +\int d^{4} x \int d^{4} y f^{(T=0)}(x-y) \frac{\phi(x)}{f_{\phi}} F_{\mu \nu}(x) F^{\mu \nu}(y) \\
& +\int d^{4} x \int d^{4} y f^{(T \neq 0)}(x-y) \frac{\phi(x)}{f_{\phi}} \bar{F}_{\mu \nu}(x) \bar{F}^{\mu \nu}(y),
\end{aligned}
$$

where

$$
\begin{aligned}
\bar{F}_{\sigma \rho}(x) & =\tilde{\partial}_{\sigma} \tilde{A}_{\rho}(x)-\tilde{\partial}_{\rho} \tilde{A}_{\sigma}(x), \\
\tilde{\partial}_{\sigma} & =\partial_{\sigma}-u_{\sigma} \partial_{0}=\left(0, \partial_{1}, \partial_{2}, \partial_{3}\right), \\
\tilde{A}_{\rho}(x) & =\tilde{\eta}_{\rho \mu} A^{\mu}(x)=A_{\rho}(x)-A_{0}(x) u_{\rho}=\left(0, A_{1}, A_{2}, A_{3}\right), \\
f^{(T=0)}(x-y) & =\int \frac{d^{4} K}{(2 \pi)^{4}} \frac{-1}{4 K^{2}} F_{N L}^{(T=0)}(K) e^{-i K \cdot(x-y)}, \\
f^{(T \neq 0)}(x-y) & =\int \frac{d^{4} K}{(2 \pi)^{4}} \frac{1}{4 \vec{k}^{2}} F_{T}^{(T \neq 0)}(\omega, \vec{k}) e^{-i K \cdot(x-y)} .
\end{aligned}
$$

We readily see that at $T=0$, the first term in eq. (3.14) corresponds to the electromagnetic scale anomaly in the vacuum, which induces the tadpole term in the effective potential viewed as a Ginzburg-Landau description. The second term of the nonlocal interaction vanishes at the low energy limit, so that it does not contribute to the effective potential describing the chiral phase transition. On the other hand, the third term corresponding to the thermal-correction part in eq. (3.14) does not drop out even in the low-energy limit, and includes an infrared divergence, which we regularize by introducing the cutoff $\mu_{\mathrm{IR}}$. Then, in the constant magnetic field $e B$, the thermomagnetically induced 
tadpole can be evaluated as

$$
\begin{aligned}
\int d^{4} x \int d^{4} y f^{(T \neq 0)}(x-y) \frac{\bar{\phi}}{f_{\phi}} \bar{F}_{\mu \nu}(x) \bar{F}^{\mu \nu}(y) & =\int d^{4} x \int d^{4} y f^{(T \neq 0)}(x-y) \frac{\bar{\phi}}{f_{\phi}} 2 B^{2} \\
& \approx \int d^{4} x\left[\frac{1}{2 e^{2}} \sum_{f} Q_{f}^{2} F\left(T, m_{\mathrm{dyn}}\right) \frac{\bar{\phi}}{f_{\phi}} \frac{|e B|^{2}}{\mu_{\mathrm{IR}}^{2}}\right],
\end{aligned}
$$

where

$$
\begin{aligned}
\sum_{f} Q_{f}^{2} F\left(T, m_{\mathrm{dyn}}\right) & =F_{T}^{(T \neq 0)}(\omega=0, \vec{k}=0) \\
& =\sum_{f} Q_{f}^{2} \frac{4 N_{c}}{\pi^{2}}\left[\left(m_{\mathrm{dyn}} T \tilde{a}\left(m_{\mathrm{dyn}} / T\right)-T^{2} \tilde{c}\left(m_{\mathrm{dyb}} / T\right)\right)+\frac{1}{4} m_{\mathrm{dyn}}^{2} \tilde{b}\left(m_{\mathrm{dyn}} / T\right)\right] .
\end{aligned}
$$

In a weak magnetic field regime (with $\sqrt{e B} \ll \mathcal{O}(\mathrm{GeV}$ ), much less than an ultraviolet cutoff scale for an effective model which we will work on in the later sections) the magnetic field strength $\tilde{F}_{12}=-B$ should numerically supply the infrared cutoff, i.e., $\mu_{\mathrm{IR}} \equiv \sqrt{e B}$. With this prescription applied, the right hand side of eq. (3.16) takes the form

$$
\int d^{4} x\left[\frac{1}{2 e^{2}} \sum_{f} Q_{f}^{2} F\left(T, m_{\mathrm{dyn}}\right) \frac{\bar{\phi}}{f_{\phi}}|e B|\right] .
$$

In total, the tadpole term is generated in the effective potential for the background field $\bar{\phi}$ and takes the form

$$
V_{\mathrm{eff}}^{(\mathrm{Tad})}(\bar{\phi})=-\frac{\bar{\phi}}{f_{\phi}}\left[\frac{\beta(e)}{e} B^{2}+\frac{1}{2 e^{2}} \sum_{f} Q_{f}^{2} F\left(T, m_{\mathrm{dyn}}\right)|e B|\right] .
$$

As the magnetic field gets strong, the one-loop calculation depending the magnetic field should be taken into account. However, the dynamics of quarks are governed by the lowest Landau level states polarized along the direction parallel to the magnetic field, where the transverse part of the photon polarization does not couple to quarks. Thus, the tadpole term induced by the electromagnetic scale anomaly eq. (3.19) vanishes in the strong magnetic field regime, which is most effective in the weak magnetic regime.

In eq. (3.19), the $\phi$ can be identified as the sigma meson which plays a role of the order parameter for the spontaneous chiral symmetry breaking. In terms of the linear sigma field $\Phi$, we find $\phi=\sqrt{2 \operatorname{tr}\left[\Phi^{\dagger} \Phi\right]}=\sqrt{\sigma_{0}^{2}+\pi^{2}+\cdots}$, and $\bar{\phi}=\bar{\sigma}_{0}$ when we only focus on the $\sigma_{0}$-direction, which is relevant to the chiral phase transition. Then the $\phi$-tadpole term in eq. (3.19) can be expressed as

$$
V_{\mathrm{eff}}^{(\mathrm{Tad})}\left(\bar{\sigma}_{0}\right)=-\sqrt{\frac{2}{N_{f}}} \frac{\bar{\sigma}_{0}}{f_{\pi}}\left[\frac{\beta(e)}{e} B^{2}+\frac{1}{2 e^{2}} \sum_{f} Q_{f}^{2} F\left(T, m_{\mathrm{dyn}}\right)|e B|\right],
$$


where we have used $f_{\phi}=\sqrt{N_{f} / 2} f_{\pi}$. Hence, the scale-anomaly induced-tadpole terms in eq. (3.20) give the contributions to the chiral phase transition. This thermomagnetic tadpole arises even in the chiral limit, as the magnetically induced-explicit chiral-breaking effect.

Before closing this section, we give a comment on possible contribution from the gluonic scale-anomaly, which is thought to be present all the way in the thermal QCD including the vacuum. It is known that the gluonic scale-anomaly can be introduced as the log potential form in the scale-chiral Lagrangian [35], which satisfies the low energy theorem in eq. (3.1). In the linear sigma model, however, the meson terms already saturate the low energy theorem, i.e., the scale anomaly and the partially-conserved dilatation current relation:

$$
\begin{aligned}
& \partial_{\mu} j_{D}^{\mu}=\left(T_{\mu}^{\mu}\right)_{\text {meson }}=2 \mu^{2} \operatorname{tr}\left[\Phi^{\dagger} \Phi\right]+\left(N_{f}-4\right) B\left(\operatorname{det} \Phi+\operatorname{det} \Phi^{\dagger}\right)-3 c \operatorname{tr}\left[\mathcal{M} \Phi^{\dagger}+\mathcal{M}^{\dagger} \Phi\right], \\
& \rightarrow \quad\left\langle 0\left|\left(T^{\mu}{ }_{\mu}\right)_{\text {meson }}(x)\right| \sigma_{0}(p)\right\rangle=\sqrt{\frac{N_{f}}{2}} f_{\pi} m_{\sigma}^{2} e^{-i p \cdot x} .
\end{aligned}
$$

Thereby, such a conventional log interaction term will be redundant. However, one might still think how much the log potential form like $\bar{\sigma}_{0}^{4} \log \bar{\sigma}_{0}$ is responsible for the chiral phase transition. Actually, the log potential can be rewritten as a combination of $\bar{\sigma}_{0}^{4}$ term and $\bar{\sigma}_{0}^{4+\epsilon}$ term with a small $\epsilon, \epsilon \ll 1$. Therefore, the log potential does not give any interference for the electromagnetically induced-scale anomaly tadpole term. Thus the tadpole term cannot be washed out and is intact even if we include the redundant log potential into the effective potential.

\section{A new critical endpoint: demonstration based on quark meson model in the large $N_{c}$ limit}

To explicitly see what form the tadpole takes, we employ a quark-meson model in a constant weak magnetic field. ${ }^{4}$ The model Lagrangian is built based upon renormalizable interactions among quarks and mesons allowed by the chiral invariance. It is constructed from the linear sigma model part in eq. (2.4) and the Dirac-fermion kinetic term for quarks, together with the Yukawa interaction term between quarks and mesons. The Yukawa coupling is introduced to be flavor universal, as it should be, because QCD is flavor blind:

$$
\mathcal{L}_{\text {Yukawa }}=-\sum_{q} g_{q}\left(\bar{q}_{L} \Phi q_{R}+\text { h.c. }\right) \quad \text { with } \quad g_{q} \equiv g .
$$

Thus, the quark-meson model Lagrangian goes like

$$
\mathcal{L}_{\mathrm{QM}}=\mathcal{L}_{\mathrm{LSM}}+\mathcal{L}_{\text {kin }}^{\text {quarks }}+\mathcal{L}_{\text {Yukawa }}
$$

As in the literature [38], we may work in the large $N_{c}$ limit, and only take into account the quark loops at one-loop level.

\footnotetext{
${ }^{4}$ It has been discussed [36, 37] that in a strong magnetic field regime, the quark-meson model can reproduce the reduction of the (pseudo) critical temperature of the chiral crossover and the inverse magnetic catalysis for the quark condensate, discovered on the lattice [1, 23-27].
} 
As in eq. (2.1), we take the flavor symmetric order parameter, $\bar{\sigma}_{u}=\bar{\sigma}_{d}=\bar{\sigma}_{s}=\bar{\sigma}_{0}$, though potentially non-negligible flavor breaking can be induced by the magnetic field. This prescription can be justified as follows: as it will be seen, the later concrete analysis will be based on the quark-meson model at the large $N_{c}$ limit, where among the charged particle contributions, only the quark loops via the flavor-universal Yukawa coupling in eq. (4.1) contribute to the chiral order parameter at vacuum $(T=e B=0)$. Thus, in the thermomagnetic system with massless three or two flavors, only the charge difference among three or two quarks makes the chiral order parameters flavorful, which comes with the magnetic field. Meanwhile, lattice QCD (with $2+1$ flavors) at physical point has reported small isospin breaking in the up and down quark condensates at around the chiral crossover, even in a strong magnetic field $(\sqrt{e B} \geq 1 \mathrm{GeV})$, e.g., [18]. This small flavor breaking can be applied also among up and strange quarks with a weaker magnetic field in the present analysis. Thus we simply assume the three-flavor symmetric vacuum even in the presence of magnetic field.

In the large $N_{c}$ limit, the quantum correction to the effective potential only arises from the quark one-loop calculation regularized by the dimensional regularization, which consists of the vacuum part $V_{1-\text { loop }}^{\mathrm{vac}}$ and the thermal part $V_{1-\text { loop }}^{\mathrm{T}}[38]$. By combining the quark parts with the mesonic part in eq. (2.10) and the scale-anomaly induced-tadpole term in eq. (3.20), the effective potential based on the quark-meson model is given as,

$$
V_{\text {eff }}\left(\bar{\sigma}_{0}\right)=V_{\text {tree }}\left(\bar{\sigma}_{0}\right)+V_{1-\text { loop }}^{\mathrm{vac}}\left(\bar{\sigma}_{0}\right)+V_{1-\text { loop }}^{\mathrm{T}}\left(\bar{\sigma}_{0}\right)+V_{\text {eff }}^{(\mathrm{Tad})}\left(\bar{\sigma}_{0}\right),
$$

where

$$
\begin{aligned}
V_{1-\text { loop }}^{\mathrm{vac}}= & \frac{N_{c} m_{\mathrm{dyn}}^{4}}{(4 \pi)^{2}} \sum_{f}\left[\log \frac{\Lambda^{2}}{2\left|Q_{f} B\right|}+1\right]-\frac{N_{c}}{2 \pi^{2}} \sum_{f}\left(Q_{f} B\right)^{2}\left[\zeta^{(1,0)}\left(-1, x_{f}\right)+\frac{1}{2} x_{f} \log x_{f}\right] \\
V_{1-\text { loop }}^{T}= & -N_{c} \sum_{s, f, n} \frac{\left|Q_{f} B\right| T}{\pi^{2}} \int_{0}^{\infty} d p \log \left(1+e^{-\sqrt{p^{2}+\left(M_{\mathrm{eff}}^{(f, s)}\right)^{2}} / T}\right) \\
= & -N_{c} \sum_{f} \frac{\left|Q_{f} B\right| T}{\pi^{2}} \int_{0}^{\infty} d p\left[\log \left\{1+\exp \left(-\sqrt{p^{2}+\left(m_{\mathrm{dyn}}^{(f)}\right.}\right)^{2} / T\right)\right\} \\
& \left.+2 \sum_{n=1} \log \left\{1+\exp \left(-\sqrt{p^{2}+\left(m_{\mathrm{dyn}}^{(f)}\right)^{2}+2 n\left|Q_{f} B\right| / T}\right)\right\}\right]
\end{aligned}
$$

with

$$
\begin{aligned}
M_{\mathrm{eff}}^{(f, s)} & =\sqrt{\left(m_{\mathrm{dyn}}^{(f)}\right)^{2}+\left|Q_{f} B\right|(2 n+1-s)} \\
x_{f} & =\left(m_{\mathrm{dyn}}^{(f)}\right)^{2} /\left(2\left|q_{f} B\right|\right) .
\end{aligned}
$$

$M_{\text {eff }}^{(f, s)}$ represent the effective quark masses for the spin-up/down quark, $s= \pm 1$. $\zeta(a, x)$ is the Hurwitz zeta-function, and $\Lambda$ being the renormalization scale fixed as $\Lambda=181.96 \mathrm{MeV}$ [38]. As noted above, the dynamical quark masses as well as the quark condensates are flavor universal, and are evaluated as

$$
m_{\mathrm{dyn}}^{(u)}=m_{\mathrm{dyn}}^{(d)}=m_{\mathrm{dyn}}^{(s)}=m_{\mathrm{dyn}}=2 g \bar{\Phi}
$$




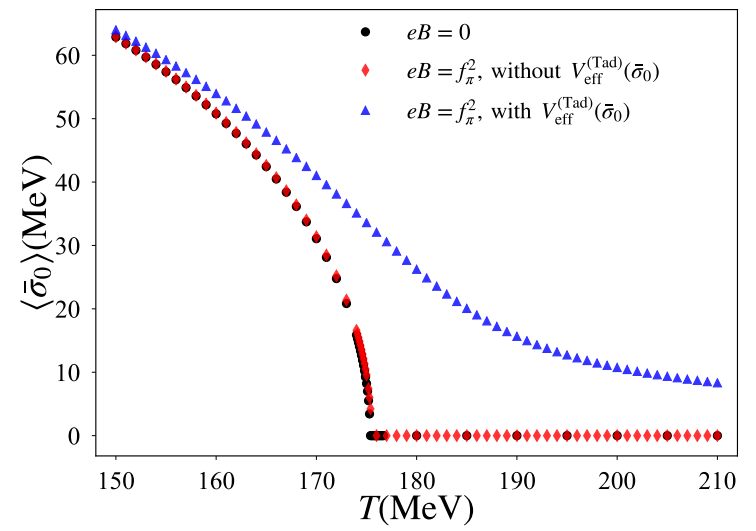

(a)

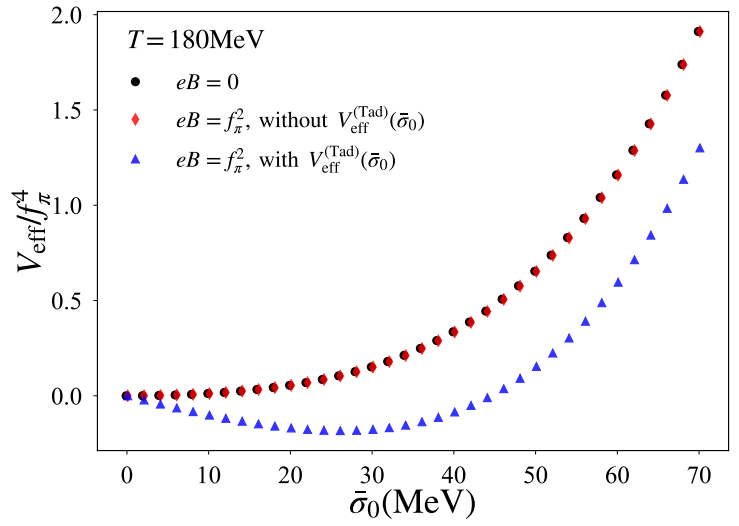

(b)

Figure 2. The chiral phase transition for massless two-flavor case, predicted from the quark-meson model in the large $N_{c}$ limit. The panel (a) shows the $T$-dependence of the chiral order parameter $\bar{\sigma}_{0}$, around the critical temperature $T_{c} \simeq 175 \mathrm{MeV}$. The panel (b) displays the deformation of the effective potential $V_{\text {eff }}$, normalized to the (forth power of) pion decay constant $f_{\pi}(\simeq 93 \mathrm{MeV})$ at vacuum. The effective potential has been normalized by subtracting $V_{\text {eff }}\left(\bar{\sigma}_{0}=0\right)$ at $T=180 \mathrm{MeV}$. Both panels monitor the $e B$ dependence in unit of $f_{\pi}^{2}$, for two cases with or without the scale anomaly-induced tadpole term included.

where $g$ is the Yukawa coupling between the quarks and the mesonic sector as in eq. (4.1). At $T=0$ and $e B=0$, we have set $m_{\mathrm{dyn}}=300 \mathrm{MeV}$ and $g=3.2258$ [38], which produces meson mass values given in appendix A.

Two plots in figure 2 exhibit a sketch of the thermomagnetic phase transition for the chiral $\mathrm{SU}(2)_{L} \times \mathrm{SU}(2)_{R}$ symmetry based on the quark-meson model with massless two flavors. As clearly seen, the scale-anomaly induced-tadpole term in eq. (3.20) makes the phase transition crossover, in somewhat a weak magnetic regime less than the (square of) chiral critical temperature $T_{c} \simeq 175 \mathrm{MeV}$ (for $\left.e B=0\right) .{ }^{5}$ This puts the milestone marked as "Crossover" at the crossed point in the $e B$ axis at $m_{s} \rightarrow \infty$, in the extended Columbia plot in figure 1.

Turning to the massless three-flavor case, we make similar plots in figure 3. In this case, the electromagnetically induced scale-anomaly tadpole competes with the U(1) axialanomaly induced cubic term. Eventually the U(1) axial-anomaly contribution wins, so that the chiral phase transition for the $\mathrm{SU}(3)_{L} \times \mathrm{SU}(3)_{R}$ symmetry keeps still of the first order, even the presence of the induced tadpole, though the chiral order parameter cannot exactly reach zero because of the tadpole. This result is marked as "1st order" in the extended Columbia plot, in figure 1. It has been noted in the literature [39] that at $T<1.5 T_{c}$ the instanton effects are not significantly suppressed. In terms of the present quark-meson model, this implies that the corresponding coupling $B_{\text {anom }}$ in eq. (2.7) for the U(1)-axial

\footnotetext{
${ }^{5}$ The value of $T_{c}$ estimated from the present quark-meson model does not agree with the result with the chiral extrapolation (only applied to the lightest two-flavors) on the lattice QCD with $2+1$ flavors, $\left.T_{c}\right|_{\text {lat. }}=132_{-6}^{+3} \mathrm{MeV}[10]$. However, this quantitative discrepancy is irrespective of our main claim in figure 1.
} 


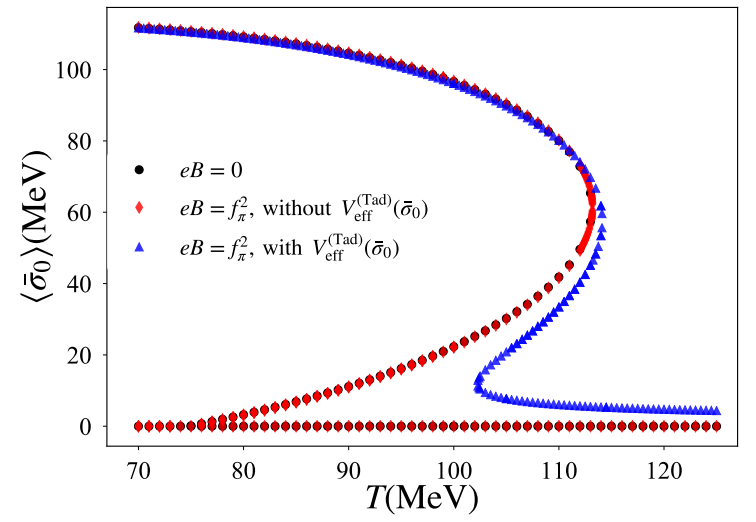

(a)

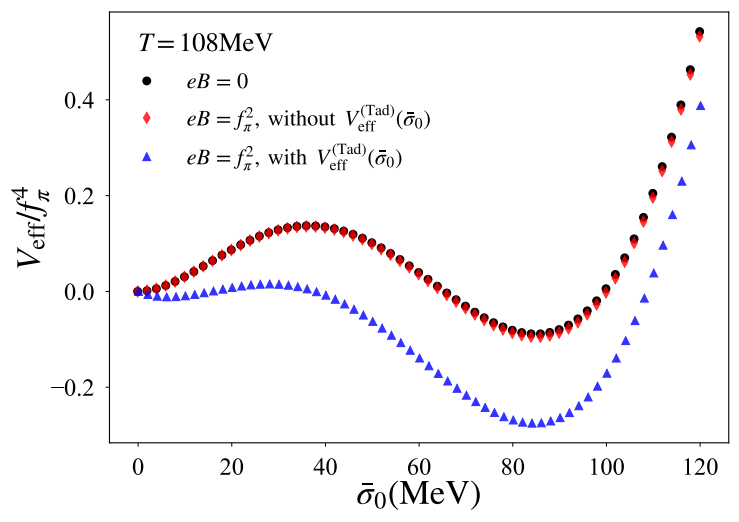

(b)

Figure 3. The same as figure 2, but for massless three flavors. The chiral critical temperature at $e B=0$ is $T_{c}=113 \mathrm{MeV}$. The effective potential $V_{\text {eff }}$ has been normalized in the same way as in figure 2 , and subtracted by the value at $\bar{\sigma}_{0}=0$ evaluated at $T=108 \mathrm{MeV}$.

anomaly term, giving the $\alpha_{3}(T, e B) \bar{\sigma}_{0}^{3}$ term in eq. (2.1), can still be effective over the possible thermal suppression at around $T=T_{c}$.

Thus, combining the explicit results in figures 2 and 3, we can conclude that in the extended Columbia plot, along the $m_{s}$ axis, there should be a critical endpoint marked as a second order phase transition - that is the "Suggested endpoint", in figure 1 dictating the critical contribution of the electromagnetic scale anomaly to the chiral phase transition.

\section{Conclusion and discussion}

In conclusion, detection of the scale anomaly in the chiral phase transition is possible: apply a weak magnetic field onto QCD with $m_{s} \rightarrow \infty$ and $m_{u}=m_{d}=0$, first observe the chiral crossover. Second, adjusting $m_{s} \searrow 0$, observe the second-order chiral phase transition, which is a new critical endpoint (See figure 1). That is the evidence of the quantum scale anomaly. This is most operative in a weak magnetic field regime.

Several comments are in order:

- Looking at figure 1 , we may have one interesting question: whether or not the second order nature persists when $m_{s}$ gets smaller along the $m_{s}$ axis. If it does, there should be a second-order phase transition line on the $m_{s}$ axis, extended from the suggested endpoint down to the first-order phase transition domain surrounded by the observed first-order point in figure 3. This is illustrated as the black-bold line on the $m_{s}$ axis, in figure 1. Here one should notice that there should also be a tri-critical point, at which the three phases (first, second, and crossover domains) overlap. It is marked by a blank square, in figure 1 . In that case, existence of the second-order phase transition line might be interpreted a remnant of the universality class of $O(4)$ on the $m_{s}$ axis, as in the Columbia plot without magnetic field [3]. 
Another second order line extended to the regime with $m_{l}>0$ is assumed to survive as in the usual Columbia plot without magnetic field, though it is yet unclear to be governed by the universality class of $Z_{2}$ (See the black-bold line separating the first order and crossover domains around the massless three-flavor regime in figure 1). If the crossover nature, confirmed at $m_{s} \rightarrow \infty$ in figure 2 , keeps until the first-order phase transition domain surrounded by the observed first-order point in figure 3 , the new critical endpoint would then become identical with the tri-critical point. Detailed analysis on identification of the tri-critical endpoint is to be pursued in another publication.

- As to the systematic expansion with respect to the magnetic field, we have applied the standard way as has been applied on lattice QCD, e.g. [25, 40]. When expanded formally in amplitudes including the vacuum polarization function of photon, the magnetic field is assumed to be small compared to a cutoff scale intrinsic to the model, which is the lattice scaling in the case of lattice QCD, and for the present quark-meson model it is an ultraviolet scale of $\mathcal{O}(1) \mathrm{GeV}$, above which mesons should dominantly be melted, or gluonic degrees of freedom should become relevant.

In eq. (3.20) we have presently ignored the next-to-leading order terms of $\mathcal{O}\left(e B^{2}\right)$ for the thermally induced tadpole part. However, such higher order corrections would naively be suppressed by a loop factor, compared to the leading order term of $\mathcal{O}(e B)$ in eq. (3.20). Even if it would not sufficiently be suppressed, and would potentially constructively enhance or destructively weaken the tadpole contribution, the presently addressed chiral-phase transition nature would not substantially be altered, as long as the magnetic field is weak enough, hence existence of the new critical endpoint would still be stable.

To be more rigorous, it is subject to nonperturbative analysis on the photon polarization function coupled to the sigma field. Recently, there have been attempts to compute the photon polarization in the weak field approximation at finite temperature [41]. It would be interesting to apply their technique described in the literature to our study, which is, however, beyond the scope of the present paper, instead deserve to another work.

- Currently, the chiral-extrapolation on lattice in absence of a magnetic field has extensively been systematically studied, and improved so much in the case with $2+1$ flavors [10]. On the other hand, there has been remarkable progress on creating small magnetic fields at the physical point, and the current lower bound is $\sim 100 \mathrm{MeV}$ [1]. It has also been worked out to calculate derivatives with respect to magnetic fields directly on the lattice, so in principle small magnetic fields become accessible [42]. Therefore, it is highly anticipated that the new critical endpoint is accessible to search on lattice QCD in near future.

- More on thermodynamical properties in thermomagnetic QCD with the electromagnetic scale anomaly will be pursued in another publication. It is also worth crosschecking our finding by the (nonperturbative) renormalization group method. 
- The presently proposed new critical endpoint is off the physical point. Extension by including a finite baryon chemical potential $\left(\mu_{B}\right)$ might kick it up at the physical point, analogously to the prospected existence of the QCD critical endpoint at the physical point in the conventional QCD phase diagram on the $\mu_{B}-T$ plane: the QCD critical endpoint at the physical point can be extended to form a critical surface when three quark masses are varied. This critical surface may also cover (or sweep) the smaller mass regime in evolving $\mu_{B}$ down to $\mu_{B}=0$, onto the Columbia plot, which is governed by the first-order domain stemming from the massless three-flavor limit [43] (See also the "1st order" domain in figure 1). Thus the existence of the QCD critical endpoint can be understood by a simple extrapolation along the critical surface with finite $\mu_{B}$, and can be interpreted as weakening of the first-order nature of the three-flavor chiral limit to the physical point on the Columbia plot [43]. This kind of critical surface might also be observed so as to link the suggested off-physical endpoint in figure 1 , to the physical point, when extended to finite $\mu_{B}$. Investigation along this possibility would also be worth pursuing.

- Beyond the chiral limit, we have also studied the pion-mass $\left(m_{\pi}\right)$ dependence on the chiral-phase transition nature, in light of lattice simulations in the future. Readers having particular concern in this research direction can refer to the result displayed in figure 4 , in appendix B.

- Our finding would also be relevant to modeling magnetized thermal QCD-like theories beyond the standard model of particle physics. For instance, it would impact gravitational wave productions addressed by a chiral phase transition in a dark/hidden QCD theory with three flavors together with a magnetic field weaker than the target QCD scale, which originates from redshifting the primordially produced one. Having one heavier flavor among three would be incompatible and cannot realize the first-order phase transition desired to create sources of gravitational waves.

At any rate, searching for the new critical endpoint is of importance, and paves a way to pioneer this frontier along with the scale anomaly, in the extended Columbia plot, figure 1.

\section{Acknowledgments}

We are grateful to Massimo D'Elia, Heng-Tong Ding and Gergely Endrödi for useful comments. This work was supported in part by the National Science Foundation of China (NSFC) under Grant No.11747308, 11975108, 12047569, and the Seeds Funding of Jilin University (S.M.). The work of A.T. was supported by the RIKEN Special Postdoctoral Researcher program and partially by JSPS KAKENHI Grant Number JP20K14479.

\section{A Meson masses in linear sigma model}

In this appendix, we list the mass formulae of linear-sigma model mesons at tree level (based on the model Lagrangian eq. (2.4)), which are used to fix the model parameters in 
the main text. The mass formulae at the tree-level will still be available in the large $N_{c}$ limit, where only the quark loop effects are taken into account.

For the two-flavor case, the meson masses are:

$$
\begin{aligned}
m_{\pi}^{2} & =\mu^{2}+\frac{1}{2} \lambda_{1} f_{\pi}^{2} \\
& =\sum_{f} c m_{f} \frac{1}{f_{\pi}}, \\
m_{\sigma}^{2} & =\mu^{2}+\frac{3}{2} \lambda_{1} f_{\pi}^{2} .
\end{aligned}
$$

Here, we have used the stationary condition in eq. (2.11). In addition, $B\left(\lambda_{2}\right)$ has been absorbed into $\mu^{2}\left(\lambda_{1}\right)$, as noted above. In the chiral limit $\left(m_{f} \rightarrow 0\right)$, the pion mass $m_{\pi}$ goes to zero. To implement the numerical calculation in the present paper for the two-flavor chiral limit, we have set $m_{\sigma}=800 \mathrm{MeV}$, just for a reference value as in the literature [37,38].

For the three-flavor case, meson masses are:

$$
\begin{aligned}
m_{\pi}^{2} & =\sum_{f} c m_{f} \sqrt{\frac{2}{3}} \frac{1}{f_{\pi}} \\
m_{\sigma}^{2} & =\mu^{2}-B_{\text {anom }} f_{\pi}+\frac{3}{2}\left(\lambda_{1}+3 \lambda_{2}\right) f_{\pi}^{2} . \\
m_{\eta^{\prime}}^{2} & =m_{\pi}^{2}+\frac{3}{2} B_{\text {anom }} f_{\pi} \\
m_{a_{0}}^{2} & =\frac{2}{3} m_{\eta^{\prime}}^{2}+\frac{1}{3} m_{\pi}^{2}+\lambda_{1} f_{\pi}^{2} \\
& =m_{\sigma}^{2}+m_{\eta^{\prime}}^{2}-m_{\pi}^{2}-3 \lambda_{2} f_{\pi}^{2} .
\end{aligned}
$$

For the three-flavor chiral limit analysis, meson masses have been fixed to $m_{\sigma}=650 \mathrm{MeV}$, $m_{a_{0}}=940 \mathrm{MeV}$, and $m_{\eta^{\prime}}=960 \mathrm{MeV}$, which are typical values obtained in the framework of linear sigma models [44, 45].

\section{B Chiral phase transition in three flavor symmetric limit}

Beyond the chiral limit, we have also investigated the pion-mass $\left(m_{\pi}\right)$ dependence on the chiral phase transition nature, in light of lattice simulations in the future. See figure 4, which corresponds to the $\mathrm{SU}(3)$ flavor limit (the $N_{f}=3$ diagonal line in figure 1). Varying $m_{\pi}$ from zero, we observe a critical point, $m_{\pi} \simeq 80 \mathrm{MeV}$, at which the transition nature changes from the first order to the second order, and will then be the crossover as gets closer to the physical pion mass. This places another critical endpoint in the extended Columbia plot, in figure 1 (marked as the black circular blob).

The estimated value of the critical pion mass, $m_{\pi} \simeq 80 \mathrm{MeV}$, cannot be so serious, because the present model should include the model-systematic uncertainty, which may roughly be $30 \%$ when the values of $T_{c}$ between ours and the lattice QCD result's are compared. See also footnote 5 in the main text.

Existence of this another critical endpoint seems to be trivial, because the chiral crossover for the pion mass around the physical point has already been established on 


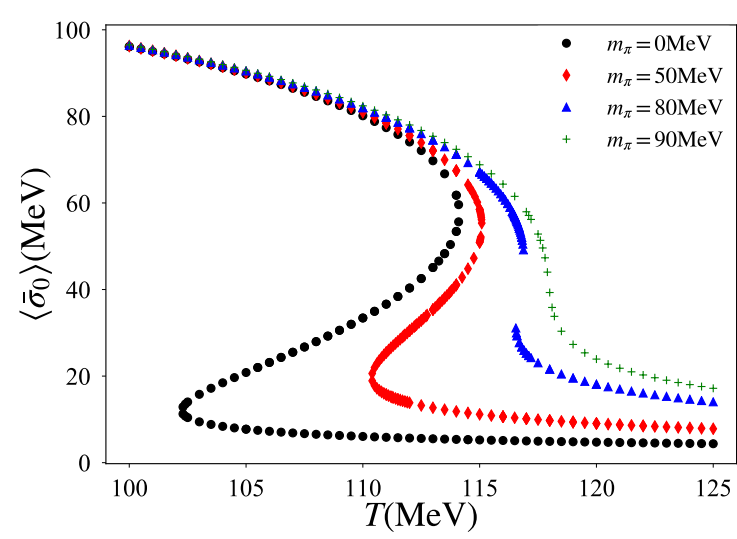

(a)

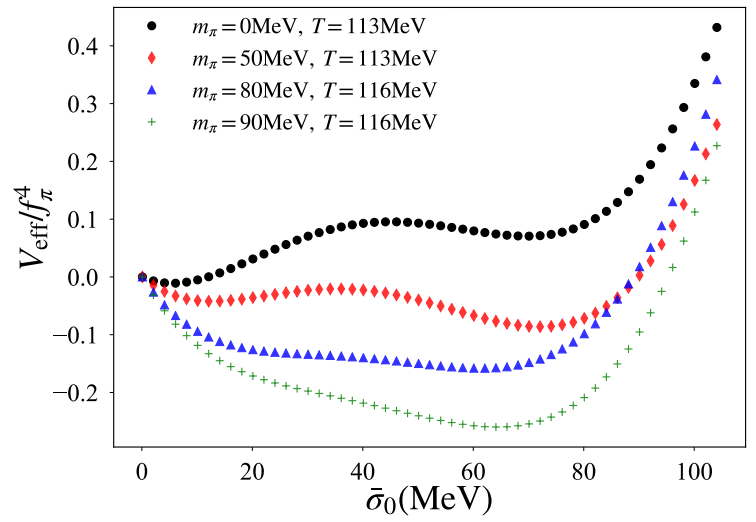

(b)

Figure 4. Sensitivity of the pion mass to the chiral phase transition in the thermomagnetic QCD at $e B=f_{\pi}^{2}$, predicted from the quark-meson model in the large $N_{c}$ limit, for the $\mathrm{SU}(3)$ flavor symmetric limit. Panel (a): the $T$-evolution of the chiral order parameter $\bar{\sigma}_{0}$. Panel (b): the corresponding deformation of the effective potential, normalized in a way similar to the ones in figures 2 and 3 in the main text.

the lattice QCD in a strong magnetic field. In this sense, we have just confirmed that the extrapolation to a weaker magnetic field regime works fine, continuously leading to the second order from the crossover. Though being such trivial, this endpoint would also be deserved to explore on the lattice QCD in the future. In contrast, the critical endpoint claimed in the main text (in the $m_{s}$ axis, arising as the interplay along the massless two and three flavor limits) is nontrivial due to the emergence of the dramatic change of the transition nature into the crossover (for two-flavor) from the first order (three-flavor).

Open Access. This article is distributed under the terms of the Creative Commons Attribution License (CC-BY 4.0), which permits any use, distribution and reproduction in any medium, provided the original author(s) and source are credited.

\section{References}

[1] G.S. Bali et al., The QCD phase diagram for external magnetic fields, JHEP 02 (2012) 044 [arXiv:1111.4956] [INSPIRE].

[2] R.D. Pisarski and F. Wilczek, Remarks on the Chiral Phase Transition in Chromodynamics, Phys. Rev. D 29 (1984) 338 [InSPIRE].

[3] F.R. Brown et al., On the existence of a phase transition for QCD with three light quarks, Phys. Rev. Lett. 65 (1990) 2491 [INSPIRE].

[4] G. Cossu et al., Finite temperature study of the axial U(1) symmetry on the lattice with overlap fermion formulation, Phys. Rev. D $8 \mathbf{7}$ (2013) 114514 [Erratum ibid. $8 \mathbf{8}$ (2013) 019901] [arXiv: 1304.6145] [INSPIRE].

[5] A. Tomiya et al., Evidence of effective axial U(1) symmetry restoration at high temperature QCD, Phys. Rev. D 96 (2017) 034509 [Addendum ibid. 96 (2017) 079902] [arXiv: 1612.01908] [INSPIRE]. 
[6] S. Aoki, Y. Aoki, G. Cossu, H. Fukaya, S. Hashimoto, T. Kaneko, C. Rohrhofer and K. Suzuki. Study of axial U(1) anomaly at high temperature with lattice chiral fermions, Phys. Rev. D 103 (2021) 074506.

[7] НотQCD collaboration, The chiral transition and $\mathrm{U}(1)_{A}$ symmetry restoration from lattice QCD using Domain Wall Fermions, Phys. Rev. D 86 (2012) 094503 [arXiv:1205.3535] [INSPIRE].

[8] M.I. Buchoff et al., QCD chiral transition, U(1)A symmetry and the Dirac spectrum using domain wall fermions, Phys. Rev. D 89 (2014) 054514 [arXiv: 1309.4149] [InSPIRE].

[9] T. Bhattacharya et al., QCD Phase Transition with Chiral Quarks and Physical Quark Masses, Phys. Rev. Lett. 113 (2014) 082001 [arXiv:1402.5175] [InSPIRE].

[10] НотQCD collaboration, Chiral Phase Transition Temperature in (2+1)-Flavor QCD, Phys. Rev. Lett. 123 (2019) 062002 [arXiv: 1903.04801] [INSPIRE].

[11] H.T. Ding, S.T. Li, Swagato Mukherjee, A. Tomiya, X.D. Wang and Y. Zhang, Correlated Dirac eigenvalues and axial anomaly in chiral symmetric QCD, Phis. Rev. Lett. 126 (2021) 082001.

[12] S. Aoki, H. Fukaya and Y. Taniguchi, Chiral symmetry restoration, eigenvalue density of Dirac operator and axial U(1) anomaly at finite temperature, Phys. Rev. D 86 (2012) 114512 [arXiv: 1209.2061] [INSPIRE].

[13] T. Sato and N. Yamada, Linking $\mathrm{U}(2) \times \mathrm{U}(2)$ to O(4) model via decoupling, Phys. Rev. D 91 (2015) 034025 [arXiv: 1412.8026] [INSPIRE].

[14] Y. Nakayama and T. Ohtsuki, Bootstrapping phase transitions in QCD and frustrated spin systems, Phys. Rev. D 91 (2015) 021901 [arXiv:1407.6195] [InSPIRE].

[15] A. Bazavov et al., Chiral phase structure of three flavor QCD at vanishing baryon number density, Phys. Rev. D 95 (2017) 074505 [arXiv:1701.03548] [INSPIRE].

[16] X.-Y. Jin, Y. Kuramashi, Y. Nakamura, S. Takeda and A. Ukawa, Critical point phase transition for finite temperature 3-flavor QCD with non-perturbatively $O($ a) improved Wilson fermions at $N_{\mathrm{t}}=10$, Phys. Rev. D 96 (2017) 034523 [arXiv:1706.01178] [InSPIRE].

[17] T.D. Cohen and N. Yamamoto, New critical point for QCD in a magnetic field, Phys. Rev. D 89 (2014) 054029 [arXiv: 1310.2234] [INSPIRE].

[18] G. Endrodi, Critical point in the QCD phase diagram for extremely strong background magnetic fields, JHEP 07 (2015) 173 [arXiv: 1504.08280] [INSPIRE].

[19] D.E. Kharzeev, L.D. McLerran and H.J. Warringa, The Effects of topological charge change in heavy ion collisions: 'Event by event P and CP-violation', Nucl. Phys. A 803 (2008) 227 [arXiv: 0711.0950] [INSPIRE].

[20] M. D'Elia, Lattice QCD Simulations in External Background Fields, Lect. Notes Phys. 871 (2013) 181 [arXiv:1209.0374] [INSPIRE].

[21] D.E. Kharzeev, J. Liao, S.A. Voloshin and G. Wang, Chiral magnetic and vortical effects in high-energy nuclear collisions - A status report, Prog. Part. Nucl. Phys. 88 (2016) 1 [arXiv: 1511.04050] [INSPIRE].

[22] G. Endrödi, QCD in magnetic fields: from Hofstadter's butterfly to the phase diagram, PoS LATTICE2014 (2014) 018 [arXiv:1410.8028] [INSPIRE]. 
[23] V.G. Bornyakov, P.V. Buividovich, N. Cundy, O.A. Kochetkov and A. Schäfer, Deconfinement transition in two-flavor lattice $Q C D$ with dynamical overlap fermions in an external magnetic field, Phys. Rev. D 90 (2014) 034501 [arXiv:1312.5628] [inSPIRE].

[24] G.S. Bali, F. Bruckmann, G. Endrödi, S.D. Katz and A. Schäfer, The QCD equation of state in background magnetic fields, JHEP 08 (2014) 177 [arXiv: 1406.0269] [INSPIRE].

[25] A. Tomiya, H.-T. Ding, X.-D. Wang, Y. Zhang, S. Mukherjee and C. Schmidt, Phase structure of three flavor QCD in external magnetic fields using HISQ fermions, PoS LATTICE2018 (2019) 163 [arXiv:1904.01276] [INSPIRE].

[26] M. D'Elia, F. Manigrasso, F. Negro and F. Sanfilippo, QCD phase diagram in a magnetic background for different values of the pion mass, Phys. Rev. D 98 (2018) 054509 [arXiv: 1808.07008] [INSPIRE].

[27] G. Endrodi, M. Giordano, S.D. Katz, T.G. Kovács and F. Pittler, Magnetic catalysis and inverse catalysis for heavy pions, JHEP 07 (2019) 007 [arXiv: 1904.10296] [INSPIRE].

[28] M. Kobayashi and T. Maskawa, Chiral symmetry and eta-x mixing, Prog. Theor. Phys. 44 (1970) 1422 [INSPIRE].

[29] M. Kobayashi, H. Kondo and T. Maskawa, Symmetry breaking of the chiral $\mathrm{U}(3) \times \mathrm{U}(3)$ and the quark model, Prog. Theor. Phys. 45 (1971) 1955 [InSPIRE].

[30] G. 't Hooft, Symmetry Breaking Through Bell-Jackiw Anomalies, Phys. Rev. Lett. 37 (1976) 8 [inSPIRE].

[31] G. 't Hooft, Computation of the Quantum Effects Due to a Four-Dimensional Pseudoparticle, Phys. Rev. D 14 (1976) 3432 [Erratum ibid. 18 (1978) 2199] [InSPIRE].

[32] D.A. Clarke, O. Kaczmarek, F. Karsch and A. Lahiri, Polyakov Loop Susceptibility and Correlators in the Chiral Limit, PoS LATTICE2019 (2020) 194 [arXiv:1911.07668] [INSPIRE].

[33] H.A. Weldon, Covariant Calculations at Finite Temperature: The Relativistic Plasma, Phys. Rev. D 26 (1982) 1394 [INSPIRE].

[34] K. Ahmed and S.S. Masood, Vacuum polarization at finite temperature and density in QED, Annals Phys. 207 (1991) 460 [inSPIRE].

[35] G.E. Brown and M. Rho, Scaling effective Lagrangians in a dense medium, Phys. Rev. Lett. 66 (1991) 2720 [INSPIRE].

[36] A. Ayala, M. Loewe and R. Zamora, Inverse magnetic catalysis in the linear sigma model with quarks, Phys. Rev. D 91 (2015) 016002 [arXiv:1406.7408] [InSPIRE].

[37] J.O. Andersen, W.R. Naylor and A. Tranberg, Inverse magnetic catalysis and regularization in the quark-meson model, JHEP 02 (2015) 042 [arXiv:1410.5247] [INSPIRE].

[38] J.O. Andersen and R. Khan, Chiral transition in a magnetic field and at finite baryon density, Phys. Rev. D 85 (2012) 065026 [arXiv:1105.1290] [InSPIRE].

[39] R.D. Pisarski and F. Rennecke, Multi-instanton contributions to anomalous quark interactions, Phys. Rev. D 101 (2020) 114019 [arXiv:1910.14052] [INSPIRE].

[40] M. D'Elia and F. Negro, Chiral Properties of Strong Interactions in a Magnetic Background, Phys. Rev. D 83 (2011) 114028 [arXiv:1103.2080] [INSPIRE]. 
[41] R. Ghosh, B. Karmakar and M.G. Mustafa, Soft contribution to the damping rate of a hard photon in a weakly magnetized hot medium, Phys. Rev. D 101 (2020) 056007 [arXiv: 1911.00744] [INSPIRE].

[42] G.S. Bali, G. Endrődi and S. Piemonte, Magnetic susceptibility of QCD matter and its decomposition from the lattice, JHEP 07 (2020) 183 [arXiv:2004.08778] [INSPIRE].

[43] P. de Forcrand and M. D'Elia, Continuum limit and universality of the Columbia plot, PoS LATTICE2016 (2017) 081 [arXiv:1702.00330] [INSPIRE].

[44] M. Ishida, Possible classification of the chiral scalar sigma nonet, Prog. Theor. Phys. 101 (1999) 661 [hep-ph/9902260] [INSPIRE].

[45] Y. Kuroda, M. Harada, S. Matsuzaki and D. Jido, Inverse Mass Hierarchy of Light Scalar Mesons Driven by Anomaly-Induced Flavor Breaking, PTEP 2020 (2020) 053 D02 [arXiv:1910.09146] [INSPIRE]. 